\title{
Neutron experiments to search for new spin-dependent interactions
}

\author{
Yu. N. Pokotilovski ${ }^{1}$ \\ Joint Institute for Nuclear Research \\ 141980 Dubna, Moscow region, Russia
}

\begin{abstract}
The consideration is presented of possible neutron experiments to search for new short-range spin-dependent forces. The spin-dependent nucleon-nucleon interaction between neutron and nuclei may cause different effects: phase shift of a neutron wave in neutron interferometers of different kind, in particular of the Lloyd mirror configuration, neutron spin rotation in the pseudomagnetic field, and transverse deflection of polarized neutron beam by a slab of substance. Estimates of sensitivity of these experiments are performed.
\end{abstract}

PACS: 14.80.Mz; 12.20.Fv; 29.90.+r; 33.25.+k

Keywords: Axion; Long-range interactions; Neutron spin rotation.

\section{Introduction}

A number of proposals were published for the existence of new interactions coupling mass to particle spin [1, 2, 3, 4, 5]. On the other hand, there are theoretical indications that there may exist light, scalar or pseudoscalar, weakly interacting bosons. Generally the masses and the coupling of these particles to nucleons, leptons, and photons are not predicted by the proposed models. The most attractive solution of the strong CP problem is the existence of a light pseudoscalar boson - the axion [6]. Current algebra techniques is used to relate the masses and coupling constants of the axion and neutral pion: $m_{a}=\left(f_{\pi} m_{\pi} / f_{a}\right) \sqrt{z} /(z+1)$, where $z=m_{u} / m_{d}=0.56, f_{\pi} \approx 93 \mathrm{MeV}, m_{\pi}=$ $135 \mathrm{MeV}$, so that $m_{a} \approx\left(0.6 \times 10^{10} \mathrm{GeV} / f_{a}\right) \mathrm{meV}$. Here $f_{a}$ is the scale of Peccei-Quinn symmetry breaking.

The axion coupling to fermions has general view $g_{a f f}=C_{f} m_{f} / f_{a}$, where $C_{f}$ is the model dependent factor. The axion may have a priori mass in a very large range, namely $\left(10^{-12}<m_{a}<10^{6}\right)$ $\mathrm{eV}$. The main part of this mass range from both - low and high mass boundaries - was excluded in result of numerous experiments and constraints from astrophysical considerations [7]. Astrophysical bounds are based on some assumptions concerning the axion and photon fluxes produced in stellar plasma.

More recent constraints limit the axion mass to $\left(10^{-5}<m_{a}<10^{-3}\right) \mathrm{eV}$ with respectively very small coupling constants to quarks and photon [7].

Axion is one of the best candidates for the cold dark matter of the Universe [8]. Some recent reviews are [7, 9].

Several laboratory searches provided constraints on the product of the scalar and pseudoscalar couplings at macroscopic distances $\lambda>1 \mathrm{~mm}$. The examples of the most recent experiments are [10, 11, 12, 13].

\footnotetext{
1e-mail: pokot@nf.jinr.ru; tel: 7-49621-62790; fax: 7-49621-65429
} 
These experiments may be divided in two groups: measurement of the force between a mass and a sensor, or measurement of the frequency shift resulting from interaction between mass and a sensor (Fig. 1).

In the experiments, where neutron is used as a sensor, in addition to these two possibilities the phase shift of the neutron wave function due to spin-dependent interaction may be measured.

On the other hand there exist independent constraints on the scalar and pseudoscalar coupling constants. The first one follows from the experiments of Galileo-, Eötvös- or Cavendish-type performed with macro-bodies, in measurements of the Casimir force between closely placed macro-bodies, or from high precision particle scattering. For the interaction range of our interest: $1 \mathrm{~cm}>\lambda>10^{-4}$ $\mathrm{cm}$ the most sensitive are the measurements of Seattle and Stanford groups [14, 15, 19, 16, 17, 18]. They have searched a deviation from the Newton gravitation law

$$
U(r)=-\frac{G M m}{r}\left(1+\alpha e^{-r / \lambda}\right)
$$

where $G$ - the Newtonian gravitational constant, $M$ and $m$ are the masses of gravitating bodies.

For the monopole-monopole interaction due to exchange of the pseudo-scalar boson

$$
V_{\text {mon-mon }}(r)=\frac{g_{s}^{2}}{4 \pi} \frac{\hbar c}{r} e^{-r / \lambda}
$$

the scalar coupling constant $g_{s}$ may be inferred from the limits on $\alpha$ :

$$
g_{s}^{2}=\frac{4 \pi G m_{n}^{2} \alpha}{\hbar c} \approx 10^{-37} \alpha
$$

where $m_{n}$ is nucleon mass.

It follows from the experiments [14, 15, 16, 17, 18] that $g_{s}^{2}$ is limited by the value $10^{-40}-10^{-38}$ in the interaction range $1 \mathrm{~cm}>\lambda>10^{-4} \mathrm{~cm}$ (see also [19]). [20].

The pseudoscalar coupling constant is restricted to $g_{p}<10^{-9}$ from astrophysical considerations

Sensitivity of these experiments falls with decreasing the interaction range below $\sim 1 \mathrm{~cm}$.

The experimental limit on the monopole-dipole interaction in the $\lambda$-range $\left(10^{-4}-1\right) \mathrm{cm}$ was established in the Stern-Gerlach type experiment in which ultracold neutrons transmitted through a slit between a horizontal mirror and absorber [21]. Their limit for the value $g_{s} g_{p}$ is $\sim 10^{-15}$ at the $\lambda=10^{-2} \mathrm{~cm}$, what corresponds to the value of the monopole-dipole potential at the surface of the mirror $\sim 10^{-3} \mathrm{neV}$, which is equivalent to the magnetic field $\sim 0.2 \mathrm{G}$ in the neutron magnetic moment interaction $\mu \mathbf{H}$ with magnetic field.

Sensitivity estimates for a future ultracold neutron Stern-Gerlach type experiment were presented, which promise orders of magnitude improvements in limiting the monopole-dipole interaction [22].

Constraints on new spin-dependent interactions were also obtained from existing data on ultracold neutron depolarization in traps [23, 24].

There was also a proposal of the ultracold neutron magnetic resonance frequency shift experiment for obtaining these constraints with better precision [25]. The realization of this idea is described in [26].

Analysis of the longitudinal spin relaxation of polarized ${ }^{3} \mathrm{He}$ to constrain axion-like interactions was proposed in [27] (see also similar constraints obtained in [28] and corrections in [29]). 
It is seen that the constraints obtained and expected from further laboratory searches are weak compared to the limit on the product $g_{s} g_{p}<10^{-28}$ inferred from the above mentioned separate constraints on $g_{s}$ and $g_{p}$. Although laboratory experiments may not lead to the strongest bounds numerically, measurements made in terrestial laboratories produce the most reliable results. On the other hand the direct experimental constraints on the monopole-dipole interaction may be useful for limiting more general class of low-mass bosons irrespective to any particular theoretical model. Further the constraint on product $g_{s} g_{p}$ may be used for the limits on the coupling constant of this more general interaction.

Axions mediate a $\mathrm{P}$ - and T-reversal violating monopole-dipole interaction potential between spin and matter (polarized and unpolarized nucleons) [30]:

$$
V_{\text {mon-dip }}(\mathbf{r})=g_{s} g_{p} \frac{\hbar^{2} \boldsymbol{\sigma} \cdot \mathbf{n}}{8 \pi m_{n}}\left(\frac{1}{\lambda r}+\frac{1}{r^{2}}\right) e^{-r / \lambda},
$$

where $g_{s}$ and $g_{p}$ are dimensionless coupling constants of the scalar and pseudoscalar vertices (unpolarized and polarized particles), $m_{n}$ the nucleon mass at the polarized vertex, the nucleon spin $\mathbf{s}=\hbar \boldsymbol{\sigma} / 2, r$ is the distance between the nucleons, $\lambda=\hbar /\left(m_{a} c\right)$ is the range of the force, $m_{a}$ - the axion mass, and $\mathbf{n}=\mathbf{r} / \mathbf{r}$ is the unitary vector.

The potential between the layer of substance and the nucleon separated by the distance $x$ from the surface is:

$$
V_{\text {mon-dip }}(x)= \pm g_{s} g_{p} \frac{\hbar^{2} N \lambda}{4 m_{n}}\left(e^{-|x| / \lambda}-e^{-|x+d| / \lambda}\right)=V_{0} e^{-|x| / \lambda} \quad(d \gg \lambda),
$$

where $N$ is the nucleon density in the layer, $d$ is the layer's thickness. The "+" and "-" depends on the nucleon spin projection on $\mathrm{x}$-axis (the surface normal).

We consider different neutron experiments to search for or to constrain this interaction.

\section{Phase shift in the neutron interferometer.}

Slow neutrons with polarization normal to the surface of unpolarized matter (Fig. 2) experience shift of the phase of the wave function:

$$
\beta=\frac{2 l V(x)}{\hbar v}=g_{s} g_{p} \frac{N \lambda \lambda_{n} l}{4 \pi} e^{-x / \lambda} \longrightarrow g_{s} g_{p} \frac{N \lambda \lambda_{n} l}{4 \pi},
$$

where $\lambda_{n}$ is the neutron wavelength, $l$ is the length of the inserted sample, $x$ is the distance of the beam from the surface. We assume here that $x$ - distance of the beam from the surface is less than the interaction range $\lambda$ and both $x, \lambda \ll d$.

The sensitivity to the product of the coupling constants follows from the sensitivity to the measured phase shift:

$$
g_{s} g_{p}=\frac{4 \pi \beta}{N \lambda \lambda_{n} l}
$$

At the very low energy interferometer with $\lambda_{n}=30 \AA$ [32], $\lambda=1 \mathrm{~mm}, l=1 \mathrm{~cm}, \beta=10^{-3}$, $N=5 \times 10^{24}$, we have $g_{s} g_{p}=10^{-21}$.

\section{Neutron spin rotation.}

The neutron spin Larmor precession frequency is

$$
\omega_{L}=\frac{2 V(x)}{\hbar}=g_{s} g_{p} \frac{\hbar N \lambda}{2 m_{n}} e^{-|x| / \lambda} .
$$


Angle of spin rotation

$$
\varphi=\omega_{L} t=\omega_{L} \frac{l}{v}=g_{s} g_{p} \frac{N \lambda \lambda_{n} l}{4 \pi} e^{-|x| / \lambda},
$$

the same as in the interferometer, but the sensitivity to the $g_{s} g_{p}$ may be higher.

It follows that constraint on $g_{s} g_{p}$

$$
g_{s} g_{p}=\frac{4 \pi \varphi}{N \lambda \lambda_{n} l} e^{|x| / \lambda}
$$

At $\lambda_{n}=10 \AA, N \sim 5 \times 10^{24}, l=1 \mathrm{~m}, \lambda=1 \mathrm{~mm}$, and measurable spin rotation angle $\varphi=10^{-5}$ the limit may be obtained $g_{s} g_{p}=3 \times 10^{-23} e^{|x| / \lambda}$.

\section{Deviation of polarized neutron beam.}

The gradient of the potential of Eq. (5) is

$$
\nabla V_{m o n-\operatorname{dip}}(x)=g_{s} g_{p} \frac{\hbar^{2} N}{4 m_{n}} e^{-|x| / \lambda}
$$

and is directed along the nucleon spin direction $(\lambda \ll d)$.

The acceleration exerted by the medium on the polarized neutron is

$$
a=g_{s} g_{p} \frac{\hbar^{2} N}{4 m_{n}^{2}} e^{-|x| / \lambda} .
$$

For an estimate of the sensitivity of such an experiment we consider the experiment [31] on the search for the neutron electric charge. In this experiment the beam of cold neutrons with the wavelength $(12<\lambda<30) \AA$ passed through a strong electric field $\sim 60 \mathrm{kV} / \mathrm{cm}$, over a length of $9 \mathrm{~m}$, and possible lateral deflection of the beam was looked with a multi-slit neutron optical system when polarity of the electric field was changed alternatively.

In the search for the spin-dependent interaction the expected lateral deflection angle of the neutron beam with velocity $v$ polarized normal to the surface of the slab of substance with the length $l$

$$
\theta=\frac{v_{\perp}}{v_{\|}}=\frac{a t}{v_{\|}}=\frac{a l}{v_{\|}^{2}}=g_{s} g_{p} \frac{\hbar^{2} N l}{4 m_{n}^{2} v_{\|}^{2}},
$$

when $x \ll \lambda$. Similarly, in the neutron charge experiment

$$
\theta=\frac{v_{\perp}}{v_{\|}}=\frac{a t}{v_{\|}}=\frac{a l}{v_{\|}^{2}}=\frac{Q_{n} E l}{m_{n} v_{\|}^{2}} .
$$

From the constraints for $Q_{n}$ the sensitivity of a possible experiment for the constraints on $g_{s} g_{p}$ is:

$$
g_{s} g_{p}=\frac{4 m_{n} Q_{n} E}{\hbar^{2} N}
$$

At $Q_{n}=10^{-21} e$ [31] and $N=5 \times 10^{24} \mathrm{~cm}^{-3}$ we get $g_{s} g_{p}=10^{-22}$.

Serious requirement in this experiment is perfect magnetic shielding: the lateral deflection of the neutron beam caused by the pseudo-magnetic field at the value $g_{s} g_{p}=10^{-22}$ may be caused by the gradient of magnetic field of $\sim 10^{-5} \mathrm{G} / \mathrm{cm}$ directed normal to the slab surface. 


\section{Lloyd mirror neutron interferometer.}

The Lloyd mirror neutron interferometer, which to the author's knowledge never was tested before, can provide high sensitivity to spin-dependent forces. Fig. 3 shows the geometry.

The neutron wave vector $k^{\prime}$ in the potential $V$ is

$$
k^{\prime 2}=k^{2}-\frac{2 m V}{\hbar^{2}} ; \quad k^{\prime}=k-\frac{m V}{k \hbar^{2}},
$$

where $k$ is the neutron wave vector in the absence of potential.

The phase shift due to interaction potential of the neutron with the mirror depending on distance from the mirror is obtained by integration along trajectories $\Delta \varphi=\oint k^{\prime} d s=\varphi_{I}-\varphi_{I I}$, where $\varphi_{I}$ and $\varphi_{2}$ are phases obtained due to potential of Eq. (5) along trajectories I and II respectively:

$$
\begin{aligned}
& \varphi_{I}= k \sqrt{L^{2}+(b-a)^{2}}+\frac{m V_{0}}{k \hbar^{2} L} \sqrt{L^{2}+(b-a)^{2}} \int_{0}^{L} \exp \left[-\left(a+\frac{b-a}{L} x\right) / \lambda\right] d x= \\
&=\varphi_{\text {geom.I }}+\frac{m V_{0}}{k \hbar^{2}} \sqrt{L^{2}+(b-a)^{2}} \frac{\lambda}{b-a}\left(e^{-a / \lambda}-e^{-b / \lambda}\right)=\varphi_{\text {geom.I }}+\varphi_{\text {pot.I }}
\end{aligned}
$$

and

$$
\begin{array}{r}
\varphi_{I I}=k \sqrt{L^{2}+(b+a)^{2}}+\frac{m V_{0}}{k \hbar^{2} L} \sqrt{L^{2}+(b+a)^{2}}\left[\int_{0}^{l} \exp \left[-\left(a-\frac{b+a}{L} x\right) / \lambda\right] d x+\right. \\
\left.\int_{l}^{L} \exp \left[-\left(\frac{b+a}{L} x-a\right) / \lambda\right] d x\right]=\varphi_{\text {geom.II }}+\frac{m V_{0}}{k \hbar^{2}} \sqrt{L^{2}+(b+a)^{2}} \frac{\lambda}{b+a}\left(2-e^{-a / \lambda}-e^{-b / \lambda}\right)= \\
=\varphi_{\text {geom.II }}+\varphi_{\text {pot.II }} .
\end{array}
$$

In Eq. (18) $l=a L /(a+b)$ is $x$-coordinate of the reflection point.

With good precision $\varphi_{\text {geom.I }}-\varphi_{\text {geom.II }}=2 \mathrm{kab} / \mathrm{L}$.

For spin-dependent potential of Eq.(5) signs of potential $V_{0}$ and, respectively, the phase shift $\varphi_{\text {pot }}$ are opposite for two spin orientations in respect to the mirror surface normal.

The difference in these phase shifts, measured in the experiment

$$
\left(\varphi_{I}^{+}-\varphi_{I I}^{+}\right)-\left(\varphi_{I}^{-}-\varphi_{I I}^{-}\right)=2\left(\varphi_{I}-\varphi_{I I}\right)=\delta \varphi .
$$

For non-perfectly vertical mirror the component of gravity normal to the surface of the mirror produces the potential $V_{g r}=\mathrm{cmg} z$, where $g$ is gravitational acceleration and the coefficient $c$ depends on the angle $\theta$ between gravity vector and the mirror plane. At $\theta=1^{\prime} c \approx 3 \times 10^{-4}$. This linear potential leads to additional phase shift

$$
\delta \varphi_{g r}=\frac{c g m^{2}}{2 k \hbar^{2}(a+b)}\left[(b+a)^{2} \sqrt{L^{2}+(b-a)^{2}}-\left(b^{2}+a^{2}\right) \sqrt{L^{2}+(b+a)^{2}}\right],
$$

calculated in analogy with Eqs.(17-18).

Finally we should calculate the phase shift of the neutron wave along the beam II at the point of reflection. Neglecting imaginary part of the potential of the mirror, the amplitude of reflected wave is $r=e^{-i \Theta}$, with the phase $\Theta=2 \arccos \left(k_{n o r m} / k_{b}\right) \approx \pi-2 k_{\text {norm }} / k_{b}$, where $k_{\text {norm }}$ is normal to 
the mirror surface component of the neutron wave vector, and $k_{b}$ is the boundary wave vector of the mirror.

These two phase shifts do not depend on spin.

Fig. 4 shows the neutron wave phase shift $\delta \varphi$ at different interaction range $\lambda$. Lloyd mirror interferometer has parameters: $L=1 \mathrm{~m}, a=50 \mu \mathrm{m}$, neutron wave length $100 \AA$ (neutron velocity $40 \mathrm{~m} / \mathrm{s}$ ), and $g_{s} g_{p}=10^{-19} ; 1$ - gravitational phase shift $\delta \varphi_{g r}$ at $c=3 \times 10^{-4} ; 2-\pi$-phase shift of the ray II at reflection.

In principle, similar experiments may be performed to search for interactions of the type of Eq. (1).

Similarly to Eq. (5) additional Yukawa-type interaction of Eq. (1) yields potential between the layer of substance and the nucleon separated by the distance $x$ from the surface:

$$
U(x)=\alpha 2 \pi N G \lambda^{2} m_{n}^{2} e^{-|x| / \lambda} .
$$

The ratio of potentials of Eqs. (21) and (5) is

$$
\frac{U}{V_{\text {mon-dip }}}=\frac{\alpha}{g_{s} g_{p}} \frac{m_{n}^{3} G \lambda}{\hbar^{2}} \approx 3 \cdot 10^{-25} \lambda(\mathrm{cm}) \frac{\alpha}{g_{s} g_{p}} .
$$

At $\lambda=0.01 \mathrm{~cm}$ we obtain $\frac{U}{V_{\text {mon-dip }}} \approx 3 \cdot 10^{-27} \frac{\alpha}{g_{s} g_{p}}$. At equal experimental sensitivity to potentials we have $\alpha \approx 3 \cdot 10^{26} g_{s} g_{p}$.

\section{References}

[1] J. Leitner and S. Okubo, Phys. Rev. 136 (1964) B1542.

[2] D. Chang, R.N. Mohapatra, and S. Nussinov, Phys. Rev. Lett. 55 (1985) 2835.

[3] C. T. Hill and G. G. Ross, Nucl. Phys. B 311 (1988) 253.

[4] P. Fayet, Class. Quant. Gravit. 13 (1996) A19.

[5] B. A. Dobrescu and I. Mocioiu, JHEP 11005 (2006); arXiv: hep-ph/0605342.

[6] S. Weinberg, Phys. Rev. Lett. 40 (1978) 223; F. Wilczek, Phys. Rev. Lett. 40 (1978) 279.

[7] C. Hangmann, H. Murayama, G. G. Raffelt, L. J. Rosenberg, and K. van Bibber, (Particle Data Group), Phys. Lett. B667 (2008) 459.

[8] P. Sikivie, Phys. Rev. Lett. 51 (1983) 1415; R. Bradley, J. Clarke, D. Kinion, L. J. Rosenberg, K. van Bibber, S. Matsuki, M. Mück, P. Sikivie, Rev. Mod. Phys. 75 (2003) 777.

[9] J. Jaeckel, A. Ringwald, arXive:1002.0329; O.K Baker, G. Cantatore, J. Jaeckel and G. Mueller, arXive:1007.1835.

[10] B.R. Heckel, E.G. Adelberger, C.E. Cramer, T.S. Cook, S. Schlamminger, and U. Schmidt, Phys. Rev. D78 (2008) 092006.

[11] Wei-Tou Ni, Shean-Shi Pan, Sien-Chi Yeh, Li-Shing Hou, and Juling Wan, Phys. Rev. Lett. 82 (1999) 2439. 
[12] R. C. Ritter, L. I. Winkler, and G. T. Gillies, Phys. Rev. Lett. 70 (1993) 701;

Wei-Tou Ni, Shean-Shi Pan, sien-Chi Yeh, Li-Shing Hou, and Juling Wan, Phys. Rev. Lett. 82 (1999) 2439.

[13] G. D. Hammond, C. C. Speake, Ch. Trenkel, A. Pulido-Patón, Phys. Rev. Lett. 98 (2007) 081101.

[14] C.D. Hoyle, D.J. Kapner, B.R. Heckel, E.G. Adelberger, J.H. Gundlach, U. Schmidt, and H.E. Swanson, Phys. Rev. D 70 (2004) 042004.

[15] D.J. Kapner, T.S. Cook, E.G. Adelberger, J.H. Gundlach, B. R.Heckel, C. D. Hoyle, and H. E. Swanson, Phys. Rev. Lett. 98 (2007) 021101.

[16] J. Chiaverini, S. J. Smullin, A. A. Geraci, D. M. Weld, and A. Kapitulnik, Phys. Rev. Lett. 90 (2003) 151101.

[17] S.J. Smullin, A.A. Geraci, D.M. Weld, J. Chiaverini, S. Holmes, and A. Kapitulnik, Phys. Rev. D72 (2005) 122001.

[18] A.A. Geraci, S.J. Smullin, D.M. Weld J. Chiaverini, and A. Kapitulnik, Phys. Rev. D 78 (2008) 022002.

[19] E.G. Adelberger, B.R. Heckel, S. Hoedl, C.D. Hoyle, D.J. Kapner, and A. Upadhye, Phys. Rev. Lett. 98 (2007) 131104.

[20] G. G. Raffelt, Phys. Rep., 333-334 593 (2000).

[21] S. Baeßler, V.V. Nesvizhevsky, K.V. Protasov, and A.Yu. Voronin, Phys. Rev. D75 (2007) 075006.

[22] S. Baeßler, V. V. Nesvizhevsky, G. Pignol, K. V. Protasov, and A. Yu. Voronin, arXiv:0902.3139v1.

[23] A. P. Serebrov, Phys. Lett. B680 (2009) 423.

[24] V. K. Ignatovich, Yu. N. Pokotilovski, Eur. Phys. Journ. C64 (2009) 19.

[25] O. Zimmer, Phys. Lett. B685 (2010) 38.

[26] A.P. Serebrov, O. Zimmer, A.K. Fomin, S.N. Ivanov, E.A. Kolomensky, I.A. Krasnoshekova, M.S. Lasakov, V.M. Lobashev, A.N. Pirozhkov, V.E. Varlamov, A.V. Vasiliev, O.M. Zherebtsov, E.B. Aleksandrov, S.P. Dmitriev, N.A. Dovator, JETP. Lett. 91 (2010) 6.

[27] Yu.N. Pokotilovski, Phys. Lett. B685 (2010) 114; arXive: 0902.1682.

[28] C.B. Fu, T.R. Gentile, and W.M. Snow, Phys. Rev. D83 (2011) 031504.

[29] A.K. Petukhov, G. Pignol, D. Jullien, and K.H. Andersen, Phys. Rev. Lett., 105 (2010) 170401;

A.K. Petukhov, G. Pignol, R. Golub, arXiv: 1103.1770.

[30] J. E. Moody, F. Wilczek, Phys. Rev., D30, 130 (1984). 
[31] J. Baumann, R. Gäler, J. Kalus, W. Mampe, Phys. Rev. D37 (1988) 3107.

[32] K. Eder, M. Gruber, A. Zeilinger, R. Gäler, W. Mampe, W. Drexel, Nucl. Instr. Meth. A284 (1989) 171;

G. van der Zouw, M. Weber, J. Felber, R. Gäler, A. Zeilinger, Nucl. Instr. Meth. A440 (2000) 568 ;

M. Hino, S. Tasaki, Y. Kawabata, T. Ebisawa, P. Geltenbort, T. Brenner, J.S. Butterworth, R. Gäler, N. Achiwa, M. Utsuro, Physica A335 (2003) 230;

Ch. Pruner, M. Fally, R.A.Rupp, R.P. May, J. Vollbrandt, Nucl. Instr. Meth. A560 (2006) 598. 

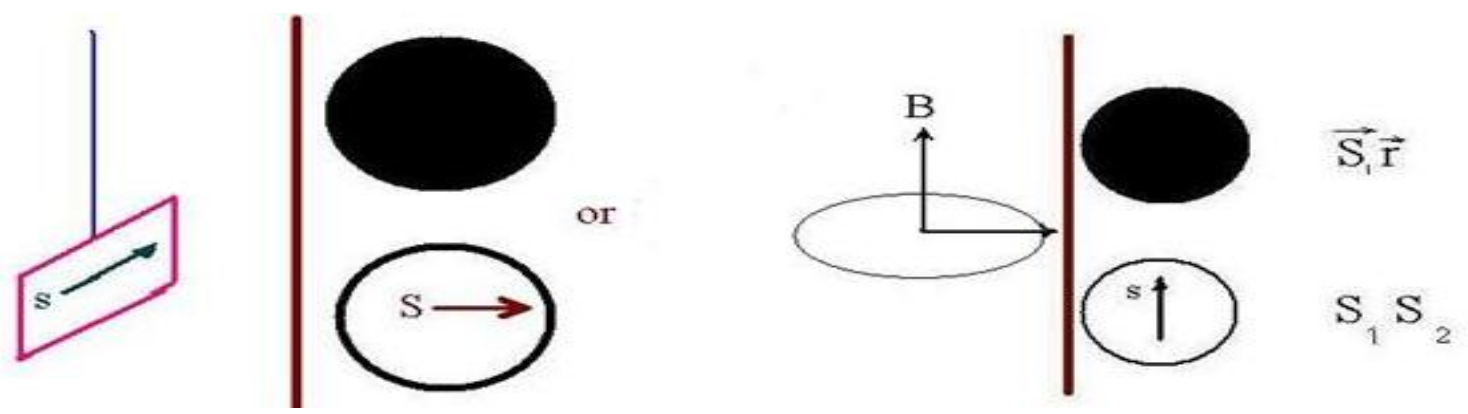

Figure 1: Scheme of macroscopic experiments to search for spin-dependent forces by: left - acceleration method, right - the frequency shift method.
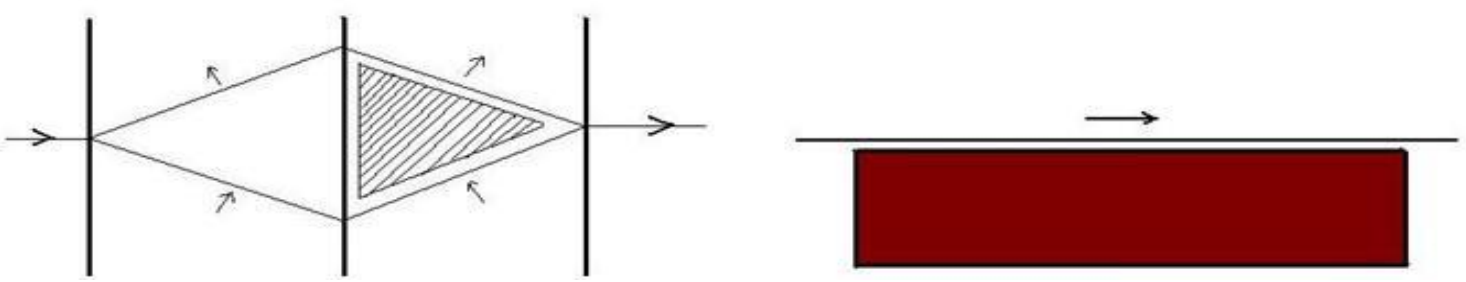

Figure 2: Left - scheme of the interferometric experiment to search for new spin-dependent forces, right - neutron spin rotation or beam deflection experiment.

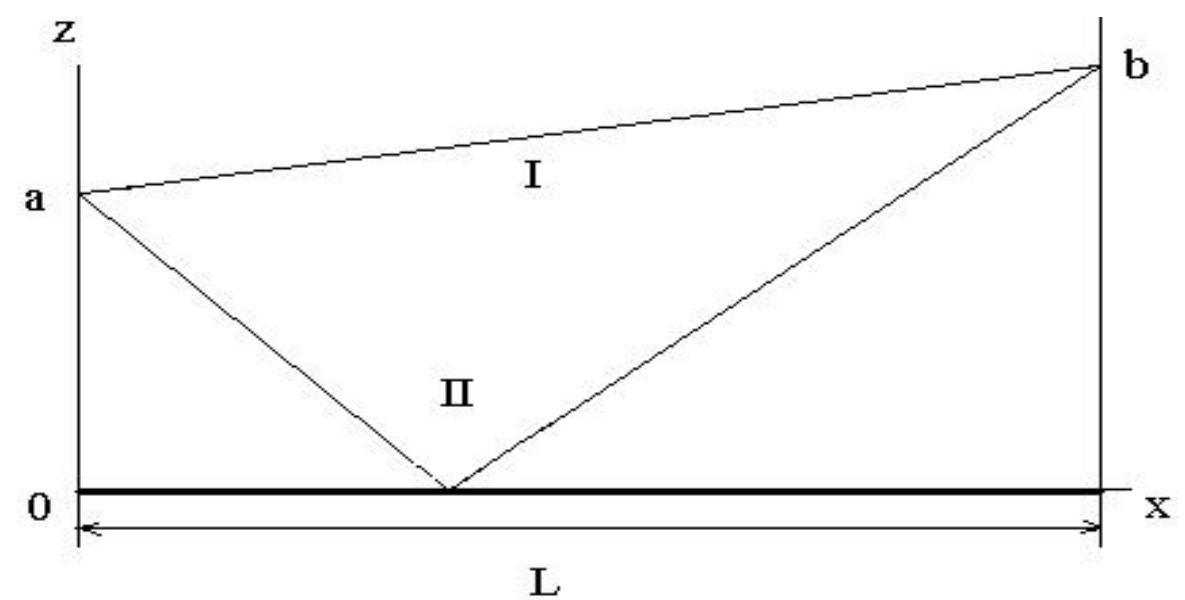

Figure 3: Lloyd neutron interferometer. Reflecting plane is vertical so that gravity effect on interference is reduced. The height of the slit above the reflecting plane is $a, L$ is the distance from the slit to the detector surface, $b$ is the height of the detector coordinate from the reflecting plane. 


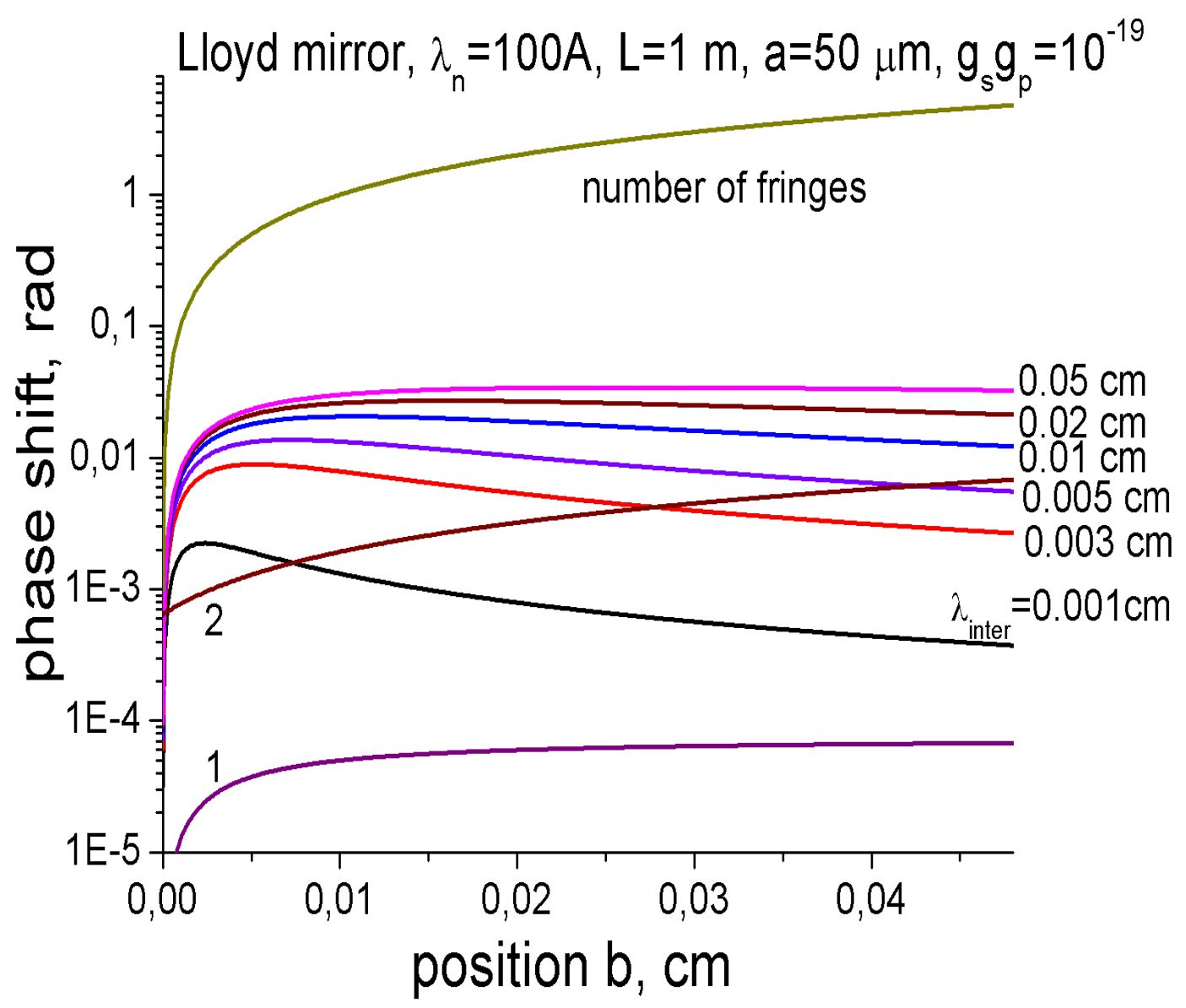

Figure 4: Neutron wave phase shift $\delta \varphi$ at different interaction range $\lambda$. Lloyd mirror interferometer has parameters: $L=1 \mathrm{~m}, a=50 \mu \mathrm{m}$, neutron wave length $100 \AA$ (neutron velocity $40 \mathrm{~m} / \mathrm{s}$ ), and $g_{s} g_{p}=10^{-19} ; 1$ - gravitational phase shift $\delta \varphi_{g r}$ at $c=3 \times 10^{-4} ; 2-\pi$-phase shift of the ray II at reflection $\left(k_{b}=10^{6} \mathrm{~cm}^{-1}\right)$. 